Translated from Teor. Mat. Fiz., 1981, v. 49, N2, pp. 210-218.

\title{
CASIMIR OPERATORS OF GROUPS OF MOTIONS OF SPACES OF CONSTANT CURVATURE
}

\begin{abstract}
N.A. Gromov
Abstract

Limit transitions are constructed between the generators (Casimir operators) of the center of the universal covering algebra for the Lie algebras of the groups of motions of $n$-dimensional spaces of constant curvature. A method is proposed for obtaining the Casimir operators of a group of motions of an arbitrary n-dimensional space of constant curvature from the known Casimir operators of the group $S O(n+1)$. The method is illustrated for the example of the groups of motions of four-dimensional spaces of constant curvature, namely, the Galileo, Poincare, Lobachevskii, de Sitter, Carroll, and other spaces.
\end{abstract}

\section{Introduction}

Lie groups and Lie algebras are widely used in many branches of theoretical physics (see, for example, [1]). Of particular interest for physics are the groups of motions of maximally homogeneous spaces - spaces of constant (in particular, zero) curvature; these have largest dimension $n(n+1) / 2$, where $n$ is the dimension of the space. The groups of Euclid, Galileo, and Poincare are well known, and their physical applications do not require additional commentaries. In [2]-[5], it is shown that the velocity space of a relativistic particle is the Lobachevskii space with group of motions $G(i, 1,1)$. Wide use has recently been made in physics [6]-[12] of the de Sitter, anti de Sitter, spherical groups and others.

Limit transitions between fundamental physical theories (classical mechanics is a limiting case of relativistic mechanics) naturally lead to the idea of limit transitions between Lie algebras and Lie groups [13]-[17]. In [18], limit transitions were constructed between the groups of motions (and their Lie algebras) of $n$-dimensional spaces of constant curvature (of these there are, as is well known [19], $3^{n}$ in total, and the number of nonisomorphic spaces is, as is shown in [20], $N_{n}=\left((3+\sqrt{5})^{n+1}-(3-\sqrt{5})^{n+1}\left(\sqrt{5} 2^{n+1}\right)^{-1}\right)$. The limit transitions of [18] enable one, if the generators and structure constants of the algebra $A G\left(j_{1}, \ldots, j_{n}\right)$ are known, to obtain the generators and structure constants of the other algebra $\tilde{A G}\left(\tilde{j}_{1}, \ldots, \tilde{j}_{n}\right)$. In particular, as the algebra $A G\left(j_{1}, \ldots, j_{n}\right)$ it is convenient to take the Lie algebra $A G(1, \ldots, 1) \equiv A G_{n}$ of the group of motions of an $n$-dimensional spherical space, which is isomorphic to the Lie algebra of the group $S O(n+1)$.

In this paper, applying the limit transition to the known [21] generators (Casimir operators) of the center of the universal covering algebra for the Lie group of $S O(n+1)$, we obtain the generators (Casimir operators) of the center of the universal covering algebra for the Lie algebra $A G\left(j_{1}, \ldots, j_{n}\right)$ of the group of motions of an arbitrary $n$-dimensional space of constant curvature. We also find transformations which carry the Casimir operators of the algebra $A G\left(j_{1}, \ldots, j_{n}\right)$ into the corresponding operators of the algebra $\tilde{A G}\left(\tilde{j}_{1}, \ldots, \tilde{j}_{n}\right)$. We illustrate the obtain formulas by the example of the Lie algebras of the groups of motions of four-dimensional spaces. 


\section{Groups of Motions of Spaces of Constant Curvature}

It is shown in [20] that an n-dimensional space of constant curvature $S\left(j_{1}, \ldots, j_{n}\right)$ can be represented locally in the form of a region on the sphere

$$
\xi_{0}^{2}+j_{1}^{2} \xi_{1}^{2}+j_{1}^{2} j_{2}^{2} \xi_{2}^{2}+\ldots+j_{1}^{2} j_{2}^{2} \ldots j_{n}^{2} \xi_{n}^{2}=1
$$

in a $(n+1)$-dimensional flat space $S\left(\iota, j_{1}, \ldots, j_{n}\right)$. The quantities $j_{k}$ take one of three values: $1, \iota, i$, where $\iota$ is a purely dual number [21], i.e., $\iota \neq 0$, but $\iota^{2}=0$. The Beltrami coordinates $x_{k}$ in the space $S\left(j_{1}, \ldots, j_{n}\right)$ are determined by the relation $x_{k}=\xi_{k} / \xi_{0}, k=1,2, \ldots, n$.

As basis generators of the group $G\left(j_{1}, \ldots, j_{n}\right)$ of motions of the space $S\left(j_{1}, \ldots, j_{n}\right)$ we choose the generators $F_{\mu \nu}$, where $\mu=0,1, \ldots, n-1, \nu=\mu+1, \mu+2, \ldots n$. Here, $F_{0 \mu}$ is the generator of transport along the $\nu$-th Beltrami coordinate axis, and it is [18]

$$
\begin{gathered}
F_{0 \nu}=-\partial_{\nu}-\left(\prod_{l=1}^{\nu} j_{l}^{2}\right) x_{\nu} \sum_{k=1}^{n} x_{k} \partial_{k}, \quad \partial_{k} \equiv \frac{\partial}{\partial x_{k}} \\
F_{\mu \nu}=-x_{\mu} \partial_{\nu}+\left(\prod_{l=\mu+1}^{\nu} j_{l}^{2}\right) x_{\nu} \partial_{\mu}
\end{gathered}
$$

is the generator of rotation in the two-dimensional plane $\left\{x_{\mu}, x_{\nu}\right\}$. We associate the pair of numbers $(\mu, \nu)$ with the number $\lambda$ in accordance with the formula

$$
\lambda=(\mu, \nu)=\nu+\mu(n-1)-\mu(\mu-1) / 2,
$$

and then the generator $F_{\mu \nu}$ can be denoted by $F_{\lambda}$, where $\lambda=1,2, \ldots n(n+1) / 2$. The structure constants of the Lie algebra $A G\left(j_{1}, \ldots, j_{n}\right)$, which are determined by the relation

$$
\left[F_{\lambda_{1}}, F_{\lambda_{2}}\right]=\sum_{\lambda=1}^{n(n+1) / 2} c_{\lambda_{1} \lambda_{2}}^{\lambda_{3}} F_{\lambda_{3}}
$$

are

$$
c_{\lambda_{1} \lambda_{2}}^{\lambda_{3}}=\left\{\begin{array}{l}
\prod_{l=\mu_{1}+1}^{\nu_{1}} j_{l}^{2}, \quad \mu_{1}=\mu_{2}, \quad \nu_{2}>\nu_{1}, \quad \lambda_{3}=\left(\nu_{1}, \nu_{2}\right), \\
\prod_{l=\mu_{2}+1}^{\nu_{2}} j_{l}^{2}, \quad \mu_{2}>\mu_{1}, \quad \nu_{1}=\nu_{2}, \quad \lambda_{3}=\left(\mu_{1}, \mu_{2}\right), \\
-1, \quad \mu_{2}>\mu_{1}, \quad \nu_{2}>\nu_{1}, \quad \nu_{1}=\mu_{2}, \quad \lambda_{3}=\left(\mu_{1}, \nu_{2}\right) .
\end{array}\right.
$$

The remaining structure constants, apart from those associated with the antisymmetry property with respect to the subscripts described in (5), are zero.

As is shown in [18], the transition from the generators and the structure constants of the group $G\left(j_{1}, \ldots, j_{n}\right)$ of motions of the unfibered ${ }^{1}$ space $S\left(j_{1}, \ldots, j_{n}\right)$ to the generators and structure constants of the group $\tilde{G}\left(\tilde{j}_{1}, \ldots, \tilde{j}_{n}\right)$ is realized in accordance with the formulas

$$
\begin{gathered}
\tilde{F}_{\lambda}=\left(\prod_{l=\mu+1}^{\nu} \tilde{j}_{l} \tilde{j}_{l}^{-1}\right) F_{\lambda}\left(\varphi x_{1}, \ldots, \varphi x_{n}\right), \\
\tilde{c}_{\lambda_{1} \lambda_{2}}^{\lambda_{3}}=c_{\lambda_{1} \lambda_{2}}^{\lambda_{3}} \prod_{i=1}^{2} \prod_{l_{i}=\mu_{i}+1}^{\nu_{i}}\left(\tilde{j}_{l i} j_{l_{i}}^{-1}\right) \prod_{l_{3}=\mu_{3}+1}^{\nu_{3}}\left(j_{l_{3}} \tilde{j}_{l_{3}}^{-1}\right),
\end{gathered}
$$

\footnotetext{
${ }^{1}$ The space $S\left(j_{1}, \ldots, j_{n}\right)$ is said to be unfibered if none of the $j_{k}, k=1,2, \ldots, n$ is equal to a purely dual number
} 
where the mapping $\varphi: S\left(j_{1}, \ldots, j_{n}\right) \rightarrow \tilde{S}\left(\tilde{j}_{1}, \ldots, \tilde{j}_{n}\right)$ is given by

$$
\varphi x_{k}=\tilde{x}_{k} \prod_{l=1}^{k} \tilde{j}_{l} j_{l}^{-1}, \quad k=1,2, \ldots, n .
$$

In particular, if we proceed from the group $G_{n}$ of motions of the $n$-dimensional spherical space isomorphic to $S O(n+1)$, Eqs.(6)-(8) simplify:

$$
\begin{gathered}
\tilde{F}_{\lambda}=\left(\prod_{l=\mu+1}^{\nu} \tilde{j}_{l}\right) F_{\lambda}\left(\varphi x_{1}, \ldots, \varphi x_{n}\right), \\
\tilde{c}_{\lambda_{1} \lambda_{2}}^{\lambda_{3}}=c_{\lambda_{1} \lambda_{2}}^{\lambda_{3}} \prod_{i=1}^{2} \prod_{l_{i}=\mu_{i}+1}^{\nu_{i}} \tilde{j}_{l i} \prod_{l_{3}=\mu_{3}+1}^{\nu_{3}} \tilde{j}_{l_{3}}^{-1}, \\
\varphi x_{k}=\tilde{x}_{k} \prod_{l=1}^{k} \tilde{j}_{l} .
\end{gathered}
$$

\section{Casimir Operators of the Group $G\left(j_{1}, \ldots, j_{n}\right)$}

We obtain the Casimir operators for the group $G\left(j_{1}, \ldots, j_{n}\right)$ from those of the group $G_{n}$ of motions of the $n$-dimensional spherical space by means of a limit transition. The Casimir operators of $S O(n+1)$, which is isomorphic to $G_{n}$, , are found in [22]. In the case of even $n=2 m$, these operators are

$$
C_{2 p}(1, \ldots, 1)=\sum_{\alpha_{1}, \ldots, \alpha_{2 p}}^{n} F_{\alpha_{1} \alpha_{2}} F_{\alpha_{2} \alpha_{3}} \ldots F_{\alpha_{2 p} \alpha_{1}}
$$

where $p=1,2, \ldots, m$, and in the case of odd $n=2 m+1$ there is added to the operators (12) the operator

$$
C^{1 / 2}(1, \ldots, 1)=\sum_{\alpha_{1}, \ldots, \alpha_{n+1}=0}^{n} \epsilon_{\alpha_{1} \alpha_{2} \ldots \alpha_{n+1}} F_{\alpha_{1} \alpha_{2}} F_{\alpha_{2} \alpha_{3}} \ldots F_{\alpha_{n} \alpha_{n+1}},
$$

where $\epsilon_{\alpha_{1} \alpha_{2} \ldots \alpha_{n+1}}$ is the completely antisymmetric unit tensor.

To make the transition to the Casimir operators $C_{2 p}\left(j_{1}, \ldots, j_{n}\right)$ and $C^{1 / 2}\left(j_{1}, \ldots, j_{n}\right)$ of the group $G\left(j_{1}, \ldots, j_{n}\right)$, we replace the generators $F$ of the group $G_{n}$ in Eqs. (12) and (13) by their expressions in terms of the generators $F^{*}$ of the group $G\left(j_{1}, \ldots, j_{n}\right)$, namely,

$$
F_{\alpha_{k} \alpha_{k+1}}=\left(\prod_{l_{k}=\mu_{k}+1}^{\nu_{k}} j_{l_{k}}^{-1}\right) F_{\alpha_{k} \alpha_{k+1}}^{*}
$$

where $\mu_{k}=\min \left(\alpha_{k}, \alpha_{k+1}\right)$, and $\nu_{k}=\max \left(\alpha_{k}, \alpha_{k+1}\right)$ and

$$
F_{\alpha_{2 p} \alpha_{1}}=\left(\prod_{l_{2 p}=\mu_{2 p}+1}^{\nu_{2 p}} j_{l_{2 p}}^{-1}\right) F_{\alpha_{2 p} \alpha_{1}}^{*}
$$

where $\mu_{2 p}=\min \left(\alpha_{2 p}, \alpha_{1}\right)$, and $\nu_{2 p}=\max \left(\alpha_{2 p}, \alpha_{1}\right)$. However, one such substitution is insufficient. Indeed, on the transition to the group of motions of a fiber bundle ${ }^{2}$ terms of the form

\footnotetext{
${ }^{2}$ The space $S\left(j_{1}, \ldots, j_{n}\right)$ is said to be $\left(k_{1}, k_{2}, \ldots, k_{r}\right)$-fibered if $j_{k_{1}}=\iota_{1}, j_{k_{2}}=\iota_{2}, \ldots, j_{k_{r}}=\iota_{r}$, where $1 \leq k_{1}<\ldots<k_{r}, 0 \leq r<n$. For $j_{1}=\iota$, we obtain flat spaces (with zero curvature); in the remaining cases, a fiber bundle is understood in the usual manner [23]. For example, $S(i, \iota)$ is a fiber bundle with base $\left\{x_{1}\right\}$ and fiber $\left\{x_{2}\right\}$.
} 
$1 / \iota$ appear in the expressions for the Casimir operators, and division by a purely dual number is not defined. Therefore, the Casimir operator $C_{2 p}$ must be multiplied by an expression which depends on $j_{1}, \ldots, j_{n}$, to make the undefined expressions disappear. Analysis of the arguments of [22] from the point of view of limit transitions shows that this expression is the lowest common denominator of the terms

$$
\prod_{k=1}^{2 p}\left(\prod_{k_{k}=\mu_{k}+1}^{\nu_{k}} j_{l_{k}}^{-1}\right)
$$

which occur in $C_{2 p}$. Similarly, the Casimir operator $C^{1 / 2}$ is multiplied by the lowest common denominator of the terms

$$
\prod_{k=1}^{n}\left(\prod_{k_{k}=\mu_{k}+1}^{\nu_{k}} j_{l_{k}}^{-1}\right)
$$

which occur in the expression for $C^{1 / 2}$. Both these lowest common denominators can be readily found. For the Casimir operator $C_{2 p}$, which is defined as the sum of the principal minors of the antisymmetric matrix formed by the generators $F_{\alpha \beta}[22]$ we obtain

$$
\prod_{k=1}^{p-1} j_{k}^{2 k} j_{n-k+1}^{2 k} \prod_{l=p}^{n-p+1} j_{l}^{2 p},
$$

and for $C^{1 / 2}$ we have

$$
j_{(n+1) / 2}^{(n+1) / 2} \prod_{k=1}^{(n-1) / 2} j_{k}^{k} j_{n-k+1}^{k} .
$$

Thus, the law of transformation of the Casimir operators on the transition from the group of motions $G_{n}$ of the spherical space to the group of motions $G\left(j_{1}, \ldots, j_{n}\right)$ has the form

$$
\begin{gathered}
C_{2 p}\left(j_{1}, \ldots, j_{n}\right)=\prod_{k=1}^{n} j_{k}^{2 p} \cdot C_{2 p}\left(F_{\lambda} \rightarrow F_{\lambda}^{*} \prod_{l=\mu+1}^{\nu} j_{l}^{-1}\right), \\
C_{2 p}^{\prime}\left(j_{1}, \ldots, j_{n}\right)=\prod_{k=1}^{p-1} j_{k}^{2 k} j_{n-k+1}^{2 k} \prod_{l=p}^{n-p+1} j_{l}^{2 p} C_{2 p}^{\prime}\left(F_{\lambda} \rightarrow F_{\lambda}^{*} \prod_{l=\mu+1}^{\nu} j_{l}^{-1}\right), \\
C^{1 / 2}\left(j_{1}, \ldots, j_{n}\right)=j_{(n+1 / 2)}^{(n+1) / 2} \prod_{k=1}^{(n+1) / 2} j_{k}^{k} j_{n-k+1}^{k} C^{1 / 2}\left(F_{\lambda} \rightarrow F_{\lambda}^{*} \prod_{l=\mu+1}^{\nu} j_{l}^{-1}\right)
\end{gathered}
$$

where

$$
F_{\lambda} \rightarrow F_{\lambda}^{*} \prod_{l=\mu+1} j_{l}^{-1}
$$

means that the generators $F$ are replaced by the generators $F^{*}$ in accordance with (14) and (15). Using (12) and (13), we write down explicit expressions for the Casimir operators of $G\left(j_{1}, \ldots, j_{n}\right)$ in terms of the generators of this group:

$$
\begin{gathered}
C_{2 p}^{\prime}\left(j_{1}, \ldots, j_{n}\right)=\sum_{\alpha_{1}, \ldots \alpha_{2 p}=0}^{n} \prod_{l=1}^{n} j_{l}^{2 p} \prod_{r=1}^{2 p} \prod_{l_{r}=\mu_{r}+1}^{\nu_{r}} j_{l_{r}}^{-1} F_{\alpha_{1} \alpha_{2}}^{*} F_{\alpha_{2} \alpha_{3}}^{*} \ldots F_{\alpha_{2 p} \alpha_{1}}^{*} \\
C^{1 / 2}\left(j_{1}, \ldots, j_{n}\right)=\sum_{\alpha_{1}, \ldots \alpha_{n+1}=0}^{n} j_{(n+1) / 2}^{(n+1) / 2} \prod_{k=1}^{(n-1) / 2} j_{k}^{k} j_{n-k+1}^{k} \prod_{r=l}^{n} \prod_{l_{r}=\mu_{r}+1}^{\nu_{r}} \times
\end{gathered}
$$




$$
\times j_{l_{r}}^{-1} \epsilon_{\alpha_{1} \ldots \alpha_{n+1}} F_{\alpha_{1} \alpha_{2}}^{*} F_{\alpha_{2} \alpha_{3}}^{*} \ldots F_{\alpha_{n} \alpha_{n+1}}^{*}
$$

The expressions (17)-(20) enable us, knowing the Casimir operators of the group of motions $G_{n}$ of the $n$-dimensional spherical space, to obtain the Casimir operators of the group of motions $G\left(j_{1}, \ldots, j_{n}\right)$ of an arbitrary $n$-dimensional space of constant curvature. An example of the application of these expressions will be considered in the fifth section.

\section{Limit Transitions Between Casimir Operators}

It is shown in [18] that a one-to-one correspondence can be established between the groups of motions $G\left(j_{1}, \ldots, j_{n}\right)$ and $\tilde{G}\left(\tilde{j}_{1}, \ldots, \tilde{j}_{n}\right)$ of unfibered and equally fibered ${ }^{3}$ spaces of constant curvature. The transition from $G$ to $\tilde{G}$ is made in accordance with (6)-(7), and the reverse transition in accordance with

$$
\begin{gathered}
F_{\lambda}=\left(\prod_{l=\mu+1}^{\nu} j_{l} \tilde{j}_{l}^{-1}\right) \tilde{F}_{\lambda}\left(\varphi^{-1} \tilde{x}_{1}, \ldots, \varphi^{-1} \tilde{x}_{n}\right) \\
c_{\lambda_{1} \lambda_{2}}^{\lambda_{3}}=\tilde{c}_{\lambda_{1} \lambda_{2}}^{\lambda_{3}} \prod_{i=1}^{2} \prod_{l_{i}=\mu_{i}+1}^{\nu_{i}}\left(j_{l} \tilde{j}_{l i}^{-1}\right) \prod_{l_{3}=\mu_{3}+1}^{\nu_{3}}\left(\tilde{j}_{l_{3}} j_{l_{3}}^{-1}\right),
\end{gathered}
$$

where the mapping $\varphi^{-1}: \tilde{S}\left(\tilde{j}_{1}, \ldots, \tilde{j}_{n}\right) \rightarrow S\left(j_{1}, \ldots, j_{n}\right)$, the inverse of (8), is

$$
\varphi^{-1} \tilde{x}_{k}=x_{k} \prod_{l=1}^{k}\left(j_{l} \tilde{j}_{l}^{-1}\right) .
$$

In addition, we can go over from the group of motions of a $\left(k_{1}, k_{2}, \ldots, k_{r}\right)$-fibered space to the group of motions of a $\left(m_{1}, m_{2}, \ldots, m_{q}\right)$-fibered space if $r<q \leq n$ and the set of numbers $\left(k_{1}, k_{2}, \ldots, k_{r}\right)$ is contained in the set $\left(m_{1}, m_{2}, \ldots, m_{q}\right)$. This transition is realized by the transformations (6) and (7).

The structure of the limit transitions between the Casimir operators is exactly the same. This can be readily seen from the following laws of transformation of the Casimir operators on the transition from $G\left(j_{1}, \ldots, j_{n}\right)$ to $\tilde{G}\left(\tilde{j}_{1}, \ldots, \tilde{j}_{n}\right)$

$$
\begin{gathered}
\tilde{C}_{2 p}^{\prime}\left(\tilde{j}_{1}, \ldots \tilde{j}_{n}\right)=\prod_{k=1}^{p-1}\left(\tilde{j}_{k} \tilde{j}_{n-k+1} j_{k}^{-1} j_{n-k+1}^{-1}\right)^{2 k} \prod_{l=p}^{n-p+1}\left(\tilde{j}_{l} j_{l}^{-1}\right)^{2 p} C_{2 p}^{\prime}\left(F_{\lambda} \rightarrow \tilde{F}_{\lambda} \prod_{l=\mu+1}^{\nu} j_{l} \tilde{j}_{l}^{-1}\right), \\
\tilde{C}^{1 / 2}\left(\tilde{j}_{1}, \ldots \tilde{j}_{n}\right)=\left(\tilde{j}_{(n+1) / 2} j_{(n+1) / 2}^{-1}\right)^{(n+1) / 2} \prod_{k=1}^{(n-1) / 2}\left(\tilde{j}_{k} \tilde{j}_{n-k+1} j_{k}^{-1} j_{n-k+1}^{-1}\right)^{k} \times \\
\times C^{1 / 2}\left(F_{\lambda} \rightarrow \tilde{F}_{\lambda} \prod_{l=\mu+1}^{\nu} j_{l} \tilde{j}_{l}^{-1}\right)
\end{gathered}
$$

and the reverse transitions from $\tilde{G}\left(\tilde{j}_{1}, \ldots, \tilde{j}_{n}\right)$ to $G\left(j_{1}, \ldots, j_{n}\right)$, which are obtained from (24) and (25) by replacing the quantities with a tilde by ones without and vice versa. The proofs are analogous to the proof of the corresponding theorems in [18].

\footnotetext{
${ }^{3}$ We say that $\left(k_{1}, k_{2}, \ldots, k_{r}\right)$-fibered and a $\left(m_{1}, m_{2}, \ldots, m_{q}\right)$-fibered space are equally fibered if $r=q$ and $k_{1}=m_{1}, k_{2}=m_{2}, \ldots, k_{r}=m_{r}$
} 


\section{Casimir Operators of the Groups $G\left(j_{1} \cdot j_{2}, j_{3}, j_{4}\right)$}

Of particular interest for physics are the groups of motions of four-dimensional spaces with constant curvature; in particular, they can serve as models of space-time. If we require of these models space to be isotropic or, equivalently, require the group $G\left(j_{1}, \ldots, j_{4}\right)$ to contain $S O(3)$ as a subgroup, we must set $j_{3}=j_{4}=1$. We shall denote the remaining nine groups for brevity by $G\left(j_{1}, j_{2}\right)$. Then $G(1,1)$, which is isomorphic to $S O(5)$, is the group of motions of the fourdimensional spherical space; $G(\iota, 1)$ is the group of motions of four-dimensional Euclidean space; $G(i, 1)$ is the group of motions of four-dimensional Lobachevskii space, $G\left(j_{1}, \iota\right)$ are the groups of motions of fibered worlds [24], i.e., space-times with absolute time. Among these, $\left(\iota_{1}, \iota\right)$ is the Galileo group, the group of motions of (flat) space-time of classical physics, and $G(1, \iota)$ and $G(i, \iota)$ are the semispherical and semi-Lobachevskii groups, ${ }^{4}$ i.e., the groups of motions of nonrelativistic space-times with positive and negative curvature, respectively. Further, $G(\iota, i)$ is the Poincare group, the group of motions of the space-time of the special theory of relativity. The de Sitter group $G(i, i)$ and the anti de Sitter group $G(1, i)$ are the groups of motions of space-times with bounded velocity of transmission of causal influences and nonvanishing constant curvature, which is negative and positive, respectively.

As is well known [22], the group $S O(5)$ has the two independent Casimir operators $C_{2}$ and $C_{4}$. These operators can be readily found for the group $G(1,1)$ isomorphic to it. We introduce new notation for the generators: $H=F_{01}, P_{k}=F_{0, k+1}, K_{k}=F_{1, k+1}, k=1,2,3, J_{1}=F_{24}, J_{2}=$ $-F_{24}, J_{3}=F_{23}$, which is in accordance with their physical meaning. Namely, $H$ is the generator of time displacement, $P_{k}$ are the generators of spatial displacements, $K_{k}$ are the generators of rotations in space-time planes (Galileo and Poincare transformations), and $J_{k}$ are the generators of spatial rotations. From formula (12) we find

$$
\begin{gathered}
C_{2}^{\prime}(1,1)=H^{2}+\mathbf{P}^{2}+\mathbf{K}^{2}+\mathbf{J}^{2}, \\
C_{4}^{\prime}(1,1)=(H \mathbf{J}-\mathbf{P} \times \mathbf{K})^{\mathbf{2}}+(\mathbf{P J})^{2}+(\mathbf{K J})^{2} .
\end{gathered}
$$

The generators $F^{*}$ of the group $G\left(j_{1}, j_{2}\right)$ are associated with the generators $F$ of the group $G(1,1)$ by the relations (9), i.e., in our case $H^{*}=j_{1} H, \mathbf{P}^{*}=j_{1} j_{2} \mathbf{P}, \mathbf{K}^{*}=j_{2} \mathbf{K}, \mathbf{J}^{*}=\mathbf{J}$. Replacing the generators $H, \mathbf{P}, \ldots$ in $(26)$ and (27) by their expressions in terms of $H^{*}, \mathbf{P}^{*}, \ldots$ and multiplying in accordance with (17) and (26) by $j_{1}^{2} j_{2}^{2}$ and (27) by $j_{1}^{2} j_{2}^{4}$, we obtain the Casimir operators of the group $G\left(j_{1}, j_{2}\right)$ :

$$
\begin{gathered}
C_{2}^{\prime}\left(j_{1}, j_{2}\right)=j_{2}^{2} H^{* 2}+\mathbf{P}^{* 2}+j_{1}^{2} \mathbf{K}^{* 2}+j_{1}^{2} j_{2}^{2} \mathbf{J}^{* 2} \\
C_{4}^{\prime}\left(j_{1}, j_{2}\right)=\left(j_{2}^{2} H^{*} \mathbf{J}^{*}-\mathbf{P}^{*} \times \mathbf{K}^{*}\right)^{2}+j_{2}^{2}\left(\mathbf{P}^{*} \mathbf{J}^{*}\right)^{2}+j_{1}^{2} j_{2}^{2}\left(\mathbf{K}^{*} \mathbf{J}^{*}\right)^{2} .
\end{gathered}
$$

For $j_{1}=j_{2}=i$, we obtain the Casimir operators of the de Sitter group (cf. [30], p. 107). Setting $j_{1}=\iota, j_{2}=i$, we obtain the Casimir operators of the Poincare group (cf. [31], Ch.2, $\S 3$; [32], §2.2). For $j_{1}=1, j_{2}=\iota$, Eqs. (28) and (29) give the Casimir operators of the semispherical and semi-Lobachevskii groups (cf. [26]). For $j_{1}=\iota_{1}, j_{2}=\iota$, we have the Casimir operators of the Galileo group (cf. [33]).

For the group $G\left(j_{1}, \ldots, j_{4}\right)$, formula $(9)$ gives the following relation between the generators: $H^{*}=j_{1} H, P_{1}^{*}=j_{1} j_{2} P_{1}, P_{2}^{*}=j_{1} j_{2} j_{3} P_{2}^{*}, P_{3}^{*}=j_{1} j_{2} j_{3} j_{4} P_{3}^{*}, K_{1}^{*}=j_{2} K_{1}, K_{2}^{*}=j_{2} j_{3} K_{2}, K_{3}^{*}=$ $j_{2} j_{3} j_{4} K_{3}, J_{1}^{*}=j_{4} J_{1}, J_{2}^{*}=j_{3} j_{4} J_{2}, J_{3}^{*}=j_{3} J_{3}$, and the Casimir operators of this group are

$$
C_{2}^{\prime}\left(j_{1}, \ldots, j_{4}\right)=j_{2}^{2} j_{3}^{3} j_{4}^{2} H^{* 2}+j_{3}^{2} j_{4}^{2} P_{1}^{* 2}+j_{4}^{2} P_{2}^{* 2}+P_{3}^{* 2}+j_{1}^{2}\left(j_{3}^{2} j_{4}^{2} K_{1}^{* 2}+j_{4}^{2} K_{2}^{* 2}+K_{3}^{* 2}\right)+
$$
[29]

${ }^{4}$ In [25] - [28], these groups are called Hooke groups. We adhere to the terminology of the earlier papers [20], 


$$
\begin{gathered}
+j_{1}^{2} j_{2}^{2}\left(j_{3}^{2} J_{1}^{* 2}+J_{2}^{* 2}+j_{4}^{2} J_{3}^{* 2}\right) \\
C_{4}^{\prime}\left(j_{1}, \ldots, j_{4}\right)=\left(j_{2}^{2} j_{3} H^{*}\left(j_{3} J_{1}^{*}, J_{2}^{*}, j_{4} J_{3}^{*}\right)-\left(\left(\mathbf{P}^{*} \times \mathbf{K}^{*}\right)_{1}, j_{3}\left(\mathbf{P}^{*} \times \mathbf{K}^{*}\right)_{2}, j_{3} j_{4}\left(\mathbf{P}^{*} \times \mathbf{K}^{*}\right)_{3}\right)\right)^{2}+ \\
j_{2}^{2}\left(j_{3}^{2} P_{1}^{*} J_{1}^{*}, P_{2}^{*} J_{2}^{*}, P_{3}^{*} J_{2}^{*}\right)^{2}+j_{1}^{2} j_{2}^{2}\left(j_{3}^{2} K_{1}^{*} J_{1}^{*}, K_{2}^{*} J_{2}^{*} K_{3}^{*} J_{3}^{*}\right)^{2}
\end{gathered}
$$

Substituting in (30) and (31) all possible combinations of the numbers $j_{1}, j_{2}, j_{3}, j_{4}$, we find the Casimir operators of the groups of motions of all four-dimensional spaces of constant curvature.

In [34] there is a description of the kinematic group (the Carroll group), which in [35] is referred to along with the semi-Lobachevskii and semispherical groups as the "most interesting of the heretical kinematic groups." This group is the group of motions of space-time with absolute space, in constrast to the Galileo group, in which the time is absolute. In our notation, the Carroll group is the group $G\left(\iota_{1}, 1,1, \iota\right)$ with a somewhat different interpretation of the generators, namely, $H=F_{04}, P_{k}=F_{0 k}, K_{1}=F_{14}, K_{2}=F_{24}, K_{3}=F_{34}, J_{1}=F_{23}, J_{2}=$ $-F_{13}, J_{3}=F_{12}$. Redenoting the generators (30) and (31) accordingly and substituting $j_{1}=$ $\iota_{1}, j_{2}=j_{3}=1, j_{4}=\iota$, we obtain the Casimir operators of the Carroll group:

$$
C_{2}^{\prime}\left(\iota_{1}, 1,1, \iota\right)=H^{2}, \quad C_{4}^{\prime}\left(\iota_{1}, 1,1, \iota\right)=(H \mathbf{J}+\mathbf{P} \times \mathbf{K})^{2},
$$

which agrees with the Casimir operators given in [34].

\section{Conclusions}

In the present paper, we have constructed limit transitions between the Casimir operators of the groups of motions of spaces of constant curvature. The structure of these limit transitions agrees with the structure of the limit transitions between the groups of motions of spaces of constant curvature investigated in [18]. The proposed limit transitions make it possible to obtain the Casimir operators of the group of motions of an arbitrary $n$-dimensional space with constant curvature from the Casimir operators of the group of motions'of $n$-dimensional spherical space, which is isomorphic to $S O(n+1)$. However, this method is not unique. One can start from the group of motions of any $n$-dimensional unfibered space of constant curvature. We have illustrated the method by the example of the groups of motions of four-dimensional spaces with constant curvature.

\section{References}

[1] E. M. Leobl (ed.), Group Theory and Its Applications, Vols. 1 and 2, Academic Press, New York $(1968,1971)$

[2] A. P. Kotel'nikov, in: In Memorial N. I. Lobachevskii, Vol.3, Kazan' (1927), pp.37-64.

[3] V. A. Fock. The Theory of Space, Time and Gravitation, Oxford (1964).

[4] N. A. Chernikov, Preprint R-723 [in Russian], JINR, Dubna (1961).

[5] N. A. Chernikov, Preprint R2-10251 [in Russian], JINR, Dubna (1976).

[6] Yu. A. Gol'fand, Zh. Eksp. Teor. Fiz., 37, 504 (1959).

[7] V. G. Kadyshevskii, Dokl. Akad. Nauk SSSR, 147, 1336 (1962). 
[8] N. A. Chernicov and E. A. Tagirov, Ann. Inst. H. Poincare, 9, 109 (1968).

[9] G. Grensing, J. Phys. A, 10, 1687 (1977).

[10] S. J. Avis, C. J. Isham, and D. Storey, Phys. Rev. D, 18, 3565 (1978).

[11] B. Zumino, Nucl. Phys. B, 127, 189 (1977).

[12] C. Fronsdal, Phys. Rev. D, 12, 3819 (1975).

[13] E. Inonu and E. P. Wigner, Proc. Natl. Acad. Sci. USA, 39, 510 (1953).

[14] A. S. Fedenko, Usp. Mat. Nauk, 12, 235 (1957).

[15] G. A. Zaitsev, Algebraic Problems of Mathematical and Theoretical Physics [in Russian], Nauka, Moscow (1974).

[16] B. A. Rozenfel'd and L. M. Karpov, Proc. Seminar on Vector and Tensor Analysis. C.XIII [in Russian], State University, Moscow (1966), pp.168-202.

[17] Ya. Kh. Lykhmus, Limit Lie Groups [in Russian], Institute of Physics and Astronomy, Estonian Academy of Sciences, Tartu (1969).

[18] N. A. Gromov, Preprint [in Russian], Komi Branch, USSR Academy of Sciences (Nauchnye Doklady, No.37), Syktyvkar (1978).

[19] I. M. Yaglom, B. A. Rozenfel'd, and E. U. Yasinskaya, Usp. Mat. Nauk, 19, 51 (1964).

[20] R. I. Pimenov, Litov. Mat. Sb., 5, 457 (1965).

[21] B. A. Rozenfel'd, Non-Euclidean Geometries [in Russian], GITTL, Moscow (1956).

[22] I. M. Gel'fand, Mat. Sb., 26 (68), 103 (1950).

[23] N. Bourbaki, Differentiable and Analytic Manifolds. Summary of Results [Russian translation], Mir, Moscow (1975), §6.1.

[24] N. A. Gromov, Preprint [in Russian], Koma Branch, USSR Academy of Sciences (Nauchnye Doklady, No. 51), Syktyvkar (1979).

[25] H. Bacry and J.-M. Levy-Leblond, J. Math. Phys., 9, 1605 (1968).

[26] J.-R. Derome and J.-A. Dubois, Nuovo Cimento , 9, 351 (1972).

[27] J.-G. Dubois, Nuovo Cimento B, 15, 1 (1973).

[28] P. Roman and J. Haavisto, J. Math. Phys., 17, 1664 (1976).

[29] R. I. Pimenov, Dokl. Akad. Nauk SSSR, 155, 44 (1964).

[30] F. Gursey, in: Group Theory and Elementary Particles [Russian translation edited by D. Ivanenko], 9921.

[31] N. N. Bogolyubov, A. A. Logunov, and I. T. Todorov, Fundamentals of the Axiomatic Approach in Quantum Field Theory [in Russian], Nauka (1969). 
[32] Yu. V. Novozhilov, Introduction to the Theory of Elementary Particles [in Russian], Nauka, Moscow (1972).

[33] J.-M. Levy-Leblond, Group Theory and Its Applications, Vol.2 (ed. E. M. Leobl), Academic Press New York (1971), p.221.

[34] J.-M. Levy-Leblond, Ann. Inst. H. Poincare, A3, 1 (1965).

[35] F. J. Dyson, Usp. Mat. Nauk, 35, 171 (1980) (Russian translation). 